\title{
The selection of judgment categories in color discrimination'
}

Michael H. Siegel

US ARMY EDGEWOOD ARSENAL CHEMICAL RESEARCH AND DEVELOPMENT LABORATORIES

\begin{abstract}
An experiment was performed to investigate the influence of some response categories upon observers' sensitivity to color differences. Inclusion of an "equal" category or requiring two criteria instead of one led to reduced sensitivity.

\section{Introduetion}

Methodological considerations are of great importance in the measurement of color discrimination ability. It has been demonstrated, for instance, that different psychophysical methods will lead to different results and that the method of constant stimulus differences is superior to both the methods of limits and the method of adjustments for such measurements (Siegel, 1962).

Having selected the psychophysical method, the experimenter is still faced with several important methodological questions. In the method of constant stimulus differences he can instruct the observer to respond in a variety of ways. The present study was designed to investigate the influence of some alternative response categories upon observers' sensitivity scores.

\section{Apparatus}

A circular stimulus field subtending two degrees at the observer's eye was provided by a Farrand $1000 \mathrm{~mm}$. monochromator. The upper half of the field served as the standard and was set to $570 \mathrm{mu}$ with a luminance of 0.20 ft-L throughout the entire study. The lower half of the field served as the variable stimulus. Before each experimental session the observer adjusted the brightness of each of the stimuli presented in the lower half of the field to match that of the upper half. The stimulus was exposed for $0.2 \mathrm{sec}$.

\section{Dbservers}

The observers were three members of our laboratory staff. Observation was made only with the right eye. The observers employed a head and chin rest. All observers were free from color vision defects and were either emmetropic or wore corrective lenses.

\section{Conditions}

1. In the first condition five variable stimuli whose wavelength was longer than that of the standard were presented to the observer. He was required to answer yes or no to the question: "Is the variable stimulus yellower than the standard?" Immediately after this observation session, another five variable stimuli whose wavelengths were shorter than that of the standard were presented to the observer. Now the judgment required was yes or no to the question: "Is the variable stimulus greener than the standard?" Every stimulus difference was presented a total of 10 times in a random order.

2. The second condition was identical to the first condition except that the observer was permitted to use a judgment of "equal" in addition to yes or no.

3 . In the third condition the observer was presented seven to nine variable stimuli some of which were longer and some of which were shorter in wavelength than the standard stimulus. The observer was required to answer the following question: "Is the variable stimulus yellower or greener than the standard?" Again, each stimulus difference was presented a total of 10 times in a random order.

4. The fourth condition was identical to the third except that the observer was permitted to use a judgment of "equal" in addition to yellower or greener.

The order of appearance of each of the four conditions was randomized and then counterbalanced for each observer. The resulting order of these eight sessions was then repeated. This permitted each observer to experience each of the four conditions four times. Results

The standard deviation was used as the response measure. The procedure for obtaining the standard deviation has been discussed elsewhere (Siegel \& Dimmick, 1962).

The results are presented in Table 1 . The values in this table represent average standard deviations for the four observing sessions under each of the four conditions.

Table 1 shows that sensitivity was always lower with an "equal" category than without one. This held both when an "equal" category was added to the yes-no judgment of the first condition and when an "equal" category was added to the yellower-greener judgment

Table 1

Average Standard Deviation Scores for All Observers

\begin{tabular}{ccccc} 
& \multicolumn{5}{c}{ Conditions } \\
Observers & 1 & 2 & 3 & 4 \\
CH & .659 & .673 & .763 & .904 \\
JM & .562 & .679 & .586 & .723 \\
MS & .722 & .794 & .743 & .932
\end{tabular}

The values in the table are average standard deviations expressed in millimicrons. See the text for a description of the four conditions. 
of the third condition. It is also clear that for each observer the yellower-greener judgments led to poorer sensitivity than did the yes-no judgments.

\section{Discussion}

The results show quite clearly that the choice of response category does affect color discrimination sensitivity. There is clear evidence that the inclusion of an "equal" category reduced sensitivity. This finding agrees with the early studies of weight lifting (Fernberger, 1930). In addition, the present study demonstrates that forced yes-no judgment leads to greater sensitivity than does the yellower-greener judgment. Both of these results can probably be accounted for by the comparative simplicity of the judgment for the observer either when an "equal" category is omitted or when only one criterion is used. That is, a greater sensitivity was displayed whenever the number of response dimensions was reduced.

If we adopt Blackwell's criterion of sensory determinacy (Blackwell, 1952) and assume that the sensory ability is best reflected by the condition which leads to the greatest sensitivity scores, then Condition 1 should be used for such experimentation. Clearly, though, it is essential that the experimenter report the observing conditions and the categories available for the observer, for omission of this information makes intelligent comparison with similar studies impossible.

\section{References}

BLACKWELL, H. R. Studies of psychophysical methods for measuring visual thresholds. J. Opt. Soc. Amer., 1952, 42, 606-616.

FERNBERGER, S. M. The use of equality judgments in psychophysical procedures. Psychol. Rev., 1930, 37, 107-112.

SIEGEL, M. H. Discrimination of color. I. Comparison of three psychophisical methods. J. Opt. Soc. Amer., 1962, 52, 1067-1070.

SIEGEL, M. H., \& DIMMICK, F. L. Discrimination of color. II. Sensitivity as a function of spectral wavelength, 510-630 mu. J. Opt. Soc. Amer., 1962, 52, 1071-1074.

Note

1. This research was supported in part by the US Army Edgewood Arsenal Chemical Research and Development Laboratories In-House Laboratory Independent Research Program. The author is grateful to Clare Holzman and Judith Bury for their help in running the study. 